\title{
Measurement of shear wave velocities coupled with an evaluation of elasticity using ARFI elastography in diagnosis of papillary thyroid carcinoma
}

\author{
Ryuhei Okada $^{1 *}$, Masami Suzuki ${ }^{1}$, Koji Takeuchi $^{2}$, Hiroyuki Horikoshi ${ }^{2}$, Atsunobu Tsunoda ${ }^{3}$ \\ ${ }^{1}$ Department of Head and Neck Surgery, Gunma Prefectural Cancer Center, Gunma, Japan \\ ${ }^{2}$ Department of Radiological Diagnosis, Gunma Prefectural Cancer Center, Gunma, Japan \\ ${ }^{3}$ Department of Otolaryngology, Tokyo Medical and Dental University, Tokyo, Japan \\ Email: *okadoto@tmd.ac.jp
}

Received 6 October 2013; revised 6 November 2013; accepted 13 November 2013

Copyright (C) 2013 Ryuhei Okada et al. This is an open access article distributed under the Creative Commons Attribution License, which permits unrestricted use, distribution, and reproduction in any medium, provided the original work is properly cited.

\begin{abstract}
This study aimed to evaluate the diagnostic value of measuring the shear wave velocities (Vs) of thyroid nodules in diagnosis of the thyroid carcinoma. Using Virtual Touch Tissue Quantification (VTTQ) of acoustic radiation force impulse (ARFI) elastography (ACUSON S2000 $^{\circledR}$, Siemens Medical Solutions, Mountain View, CA, USA), we measured the Vs of thyroid nodules in 39 nodules from 34 patients (four males and 30 females) before surgery. Elasticity itself was also estimated by VTTI (Virtual Touch Tissue Imaging) using ARFI. The average Vs of normal thyroid tissue was $2.24 \pm 0.68 \mathrm{~m} / \mathrm{s}$. Twenty-three out of 39 nodules showed an average $2.52 \pm 1.33 \mathrm{~m} / \mathrm{s}$. However, 16 other nodules showed " $X . X X \mathrm{~m} / \mathrm{s}$ ". These 16 nodules showed either "black" or "honeycomb" patterns of elasticity in VTTI. This value suggested that the Vs of these nodules were too fast or heterogenous to measure by this device. After the surgery, the pathologies of these nodules were revealed to be papillary carcinoma (21 cases), adenomatous goiter (11), follicular carcinoma (4), follicular adenoma (2) and follicular lymphoma (1). Five papillary carcinomas were found within adenomatous goiters. Nodules from four follicular carcinomas showed average Vs of $\mathbf{1 . 9 2}$ $\pm 0.48 \mathrm{~m} / \mathrm{s}$, while two follicular adenomas and follicular lymphoma showed $2.19 \pm 0.06$ and $2.34 \mathrm{~m} / \mathrm{s}$ respectively. Nodules of adenomatous goiter showed $2.14 \pm 0.60 \mathrm{~m} / \mathrm{s}$. On the other hand, nodules from five out of 21 papillary carcinomas showed average Vs of $4.00 \pm 2.37 \mathrm{~m} / \mathrm{s}$. In the nodules of 16 papillary carcinomas, Vs were measured as "X.XX m/s" and this value was only observed in nodules of papillary car-
\end{abstract}

${ }^{*}$ Corresponding author. cinoma. These data reflected high and/or heterogenous elasticity of papillary carcinoma, in other words, the tissue of papillary carcinoma was hard and/or heterogenous. It was notable that the $\mathrm{Vs}$ value of "X.XX m/s" strongly suggested papillary carcinoma.

Keywords: ARFI; Elastography; Thyroid; Thyroid Nodule

\section{INTRODUCTION}

Palpation is a necessary diagnostic method for the clinician. Concerning the diagnosis of thyroid diseases, the hardness of the thyroid nodule is an important factor for diagnosing thyroid tumors, and a hard mass implies the presence of carcinoma. Therefore, objective evaluation of the "hardness of nodules" must be effective in diagnosing thyroid disorders.

Ultrasound examination is valuable for diagnosis of thyroid diseases. This can image not only a cross-section but also the blood flow of the thyroid gland [1-3]. Using elastography, estimations of the elasticity of thyroid tissue can also be accomplished [4-8]. Conventional elastography, however, only shows the difference in elasticity between a lesion and the surrounding tissue. For the purpose of quantitative measurement of elasticity, Virtual Touch Tissue Quantification (VTTQ) has been developed. By giving an acoustic radiation force impulse (ARFI), that is, a sound impulse to the tissue, a corresponding shear wave is developed [9]. Hard tissue shows a faster shear wave, whereas soft tissue develops a slower shear wave. So the measuring shear wave velocity (Vs), and elasticity, in other words, the hardness of a nodule can be quantitatively evaluated. Therefore, the VTTQ must have potential as a useful modality in the diagnosis 
of thyroid lesions. In this preliminary report, we introduce our experience of Vs measurement in various thyroid pathologies, and also report on the possibility in diagnosis of papillary carcinoma.

\section{MATERIALS AND METHODS}

Between April 2011 and October 2012, we experienced 34 surgical cases of nodular thyroid lesion. Prior to surgery, all cases underwent measurement of Vs using VTTQ of ARFI elastography (ACUSON S2000, Siemens Medical Solutions, Mountain View, CA, USA). Conventional ultrasound imaging was initially performed and nodules for measurement were determined. Measurements of Vs were carried out as follows: the area of measurement was decided in the nodule and a $5 \times 5 \mathrm{~mm}$ sized region of interest (ROI) was decided inside the nodule (Figure 1). Both cystic and calcified lesions were excluded from measurements. In five out of 34 patients, two solid nodules were detected and Vs were measured. Therefore, the total Vs of 39 nodules were measured. For measurement of normal controls, we also measured the normal area of these patients. These measurements were carried out several times and the average of reproducible two values was used for study. If the value showed "X.XX m/s", we re-measured several times and the value "X.XX m/s" was finally adopted.

The elasticity of these nodules was also estimated by VTTI (Virtual Touch Tissue Imaging) using ARFI. According to $\mathrm{Gu}$ et al. [10], the degrees of nodule elasticity are classified into four: softer (the nodule is whiter than the surrounding tissue), equal (similar image color to the surrounding tissue), stiffer (the nodule appears blacker than the surrounding tissue), and honeycomb (the nodule shows an alternating black and white honeycomb-like distribution). TSH, free T4, serum antithyroid peroxydase antibody and antithyroglobulin antibody were also measured. Surgery was done within three days of the examination. The pathologies of these nodules were then compared to Vs. The pathological sections of tumor were precisely compared to the ultrasound ROI by which Vs and elasticity were measured (Figure 1). This research project was approved by our IRB for research: 40524034. Details of this research were explained prior to examination and surgery. A written informed consent was obtained from all patients.

\section{RESULTS}

All patients were euthyroid, but abnormal titers of the serum antithyroid peroxydase antibody and/or antithyroglobulin antibody were detected in 18 patients. Excluding these patients, the Vs of normal thyroid tissues measured $2.24 \pm 0.68 \mathrm{~m} / \mathrm{s}$. Twenty-three out of 39 nodules showed from 0.88 to $8.14 \mathrm{~m} / \mathrm{s}$ and the average Vs was $2.52 \pm 1.33 \mathrm{~m} / \mathrm{s}$. However, 16 other nodules showed "X.XX $\mathrm{m} / \mathrm{s}$ ". This value suggested that the Vs of these nodules were too fast or heterogenous to measure by this device. In other words, these nodules were too hard or heterogenous to allow this device to measure their elasticity.

After the surgery, the pathologies of these nodules

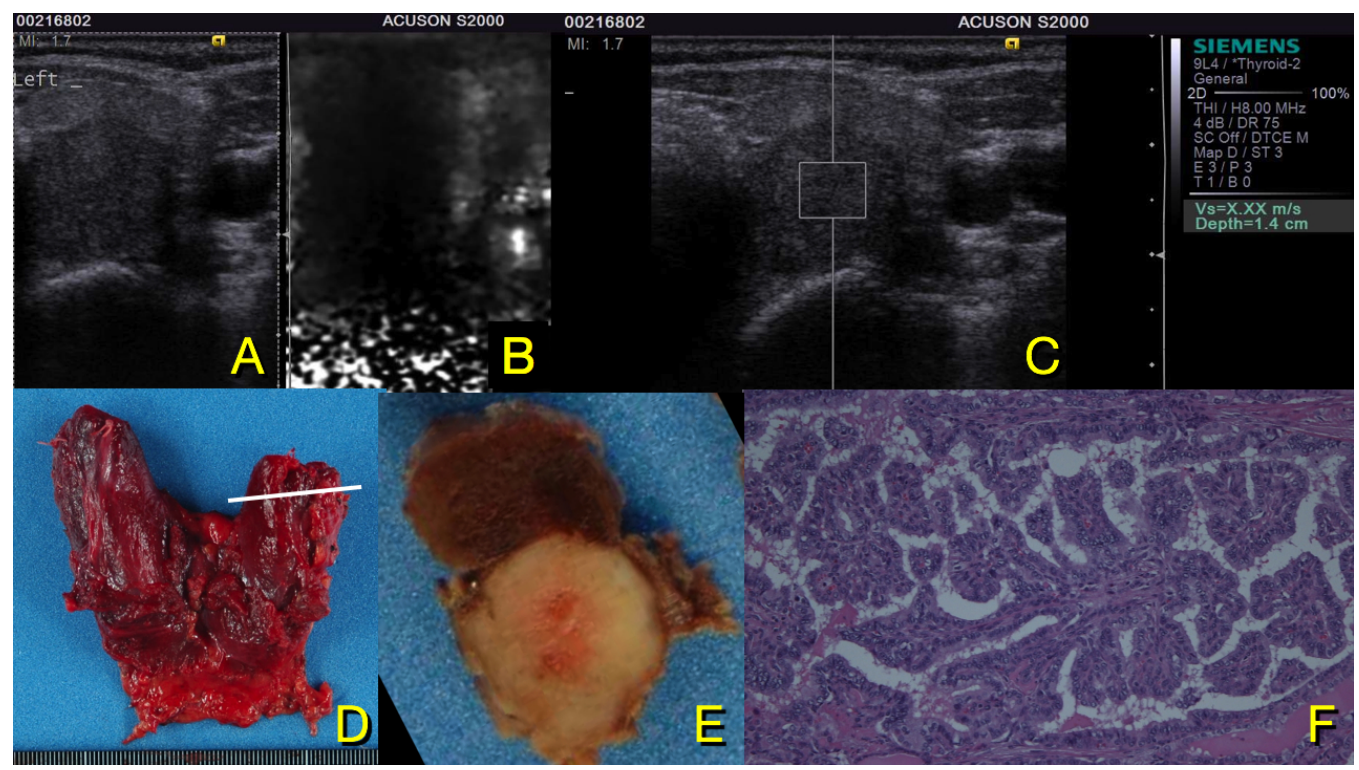

Figure 1. Measurement of shear wave velocity (Vs) for a thyroid nodule. Ultrasound examination are done at first (A), then elastologaphy (B) and measurement of Vs (C). Vs are measured in $5 \times 5 \mathrm{~mm}$ square ROI in the center of nodule. This nodule shows Vs of X.XX m/s (C). After the surgery, the section of the same nodule is made equal to the ultrasound examination ((D) and (E)) then investigated pathologically (F). This nodule A was diagnosed as papillary carcinoma. 
were revealed to be papillary carcinoma (21 cases), adenomatous goiter (11), follicular carcinoma (4), follicular adenoma (2) and follicular lymphoma (1). Five patients who had two solid nodules suffered from both adenomatous goiter and papillary carcinoma concurrently. Nodules from four follicular carcinomas showed an average Vs of $1.92 \pm 0.48 \mathrm{~m} / \mathrm{s}$, while two follicular adenomas and follicular lymphoma showed $2.19 \pm 0.06$ and $2.34 \mathrm{~m} / \mathrm{s}$ respectively. Nodules observed in adenomatous goiter showed $2.14 \pm 0.60 \mathrm{~m} / \mathrm{s}$. On the other hand, five nodules were diagnosed as papillary carcinomas, and they showed an average Vs of $4.00 \mathrm{~m} / \mathrm{s} \pm 2.37 \mathrm{~m} / \mathrm{s}$. In the nodules of 16 papillary carcinomas, Vs measured "X.XX m/s" and this value was only observed in nodules of papillary carcinoma (Table 1, Figure 2). These data reflected the high or heterogenous elasticity of papillary carcinoma, in other words, tissue of papillary carcinoma was hard or heterogenous. Concerning elasticity, nodules showing values of "X.XX m/s" appeared as "black" or "honeycomb" pattern on VTTI. This result indicated that these nodules had higher (black) or more heterogenous (honeycomb) elasticity compared to surrounding tissue. Using a value of "X.XX m/s" as an inspection standard, the sensitivity, specificity, efficiency and positive predictive value (\%) were 76.2, 100, 87.2 and 100 respectively (Table 2).

\section{DISCCUSION}

As mentioned, objective evaluation of nodule elasticity must be effective in diagnosing thyroid disorders, and VTTQ can evaluate the elasticity of thyroid tissue quantitatively. In the present study, half of the nodules were papillary carcinomas and most of these nodules showed Vs of "X.XX m/s". Apparent calcification was observed in 11 out of 21 papillary carcinomas, and although we refrained from measuring calcified areas, nevertheless 16 tumors showed "X.XX m/s". The reason for the Vs value being "X.XX m/s" is thought to be as follows. Firstly, the nodule is too hard to measure Vs appropriately. Since the maximum measurable Vs using ACUSON S2000 ${ }^{\circledR}$ is around $8.4 \mathrm{~m} / \mathrm{s}$ (personal communication: Siemens), nodules having Vs higher than $8.4 \mathrm{~m} / \mathrm{s}$ may measure Vs as "X.XX m/s". Therefore, most of the papillary carcinomas in this series might be too hard and their Vs might be higher than $8.4 \mathrm{~m} / \mathrm{s}$. The other reasons for this are that tumors have dense cellularity and/or heterogenous tissue structure. For example, the presence of microcalcification which cannot be depicted in a B-mode ultrasound image, may have a heterogenous tissue structure, and shear waves are difficult to detect in such situations.

In addition to VTTQ, that is, measurement of Vs, we observed these nodules using VTTI. VTTI depicts a nod-

Table 1. Cases of papillary carcinoma.

\begin{tabular}{|c|c|c|c|c|c|c|c|}
\hline Case & Age & Sex & Anti-TG & Anti-PO & Vs (normal area) & Vs (nodule) & VTTI \\
\hline 1 & 56 & Female & Negative & Negative & 0.91 & 8.14 & Stiffer \\
\hline 2 & 77 & Male & Positive & Negative & N.A. & X.XX & Stiffer \\
\hline 3 & 75 & Female & Negative & Negative & N.A. & X.XX & Stiffer \\
\hline 4 & 41 & Female & Positive & Negative & 2.38 & X.XX & Stiffer \\
\hline 5 & 51 & Female & Negative & Positive & 2.27 & X.XX & Stiffer \\
\hline 6 & 49 & Female & Positive & Negative & N.A. & 3.47 & honeycomb \\
\hline 7 & 54 & Female & Negative & Negative & N.A. & X.XX & Stiffer \\
\hline 8 & 54 & Female & Positive & Negative & 2.34 & X.XX & Stiffer \\
\hline 9 & 76 & Female & Positive & Positive & N.A. & X.XX & Stiffer \\
\hline 10 & 59 & Female & Negative & Negative & 1.7 & X.XX & Honeycomb \\
\hline 11 & 38 & Female & Negative & Negative & 1.77 & X.XX & Stiffer \\
\hline 12 & 55 & Female & Negative & Negative & 2.7 & X.XX & Stiffer \\
\hline 13 & 43 & Female & Negative & Negative & 2.52 & X.XX & Honeycomb \\
\hline 14 & 64 & Female & Positive & Positive & 3.06 & X.XX & Stiffer \\
\hline 15 & 58 & Female & Negative & Negative & N.A. & 2.74 & Stiffer \\
\hline 16 & 24 & Female & Negative & Negative & N.A. & 2.34 & Honeycomb \\
\hline 17 & 71 & Female & Negative & Negative & 2.04 & 3.12 & Stiffer \\
\hline 18 & 61 & Female & Negative & Negative & 1.47 & X.XX & Stiffer \\
\hline 19 & 38 & Female & Negative & Negative & 2.12 & 2.52 & Stiffer \\
\hline 20 & 68 & Male & Negative & Negative & 2.74 & X.XX & Stiffer \\
\hline 21 & 41 & Female & Negative & Negative & 1.31 & X.XX & Stiffer \\
\hline
\end{tabular}

The Vs of papillary carcinomas of the present study. Seven out of 21 cases, Vs of normal area are not shown since they showed positive antithyroid peroxydase antibody and/or antithyroglobulin antibody. N.A.: not assessed. 


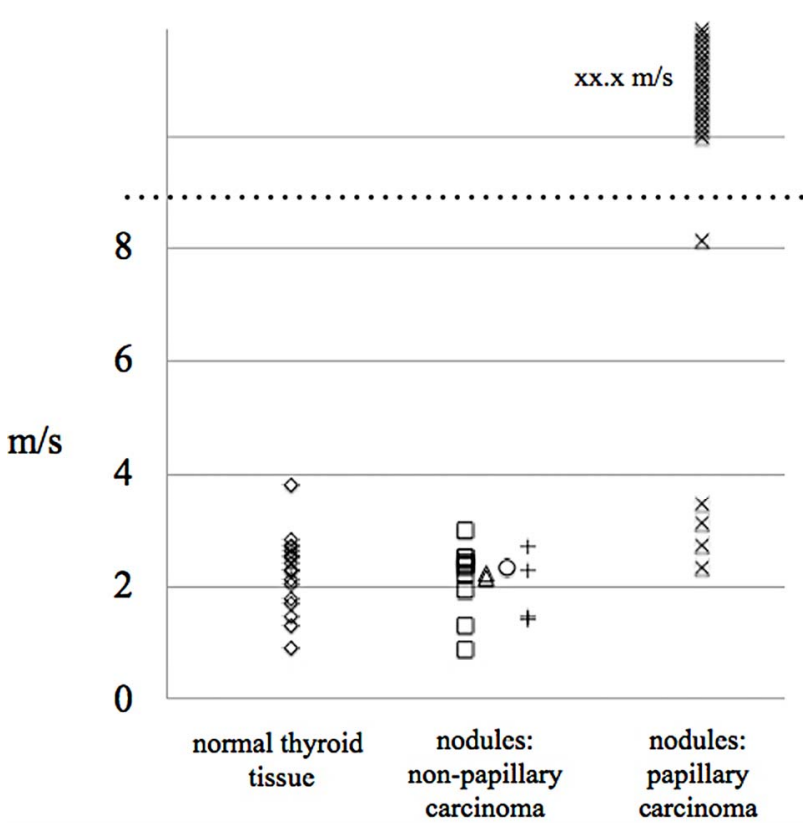

Figure 2. Distribution of velocity of thyroid nodules. The dotted line indicates the maximum measurable Vs of this device. Nodules of papillary carcinoma apparently show higher Vs compared to other nodules.

Table 2. Vs of nodules.

\begin{tabular}{ccc}
\hline Shear wave velocity & \multicolumn{2}{c}{ Pathology } \\
\hline $\mathrm{m} / \mathrm{s}$ & Papillary carcinoma Non-papillary carcinoma \\
\hline X.XX & 16 & 0 \\
Measurable $(0.88-8.14)$ & 5 & 19 \\
\hline
\end{tabular}

Contingency table of papillary carcinomas and other lesions. Using a value of X.XX m/s (possibly $>8.4 \mathrm{~m} / \mathrm{s}$ ) as an inspection specificity (\%) is 100 . In other words, if Vs show X.XX m/s, the node is highly likely to be papillary carcinoma.

ule's elasticity, and "Black" indicates higher elasticity of the nodule compared to surrounding tissue. "Honeycomb" indicates heterogenous elasticity within the nodule. Unlike conventional elastography, VTTI uses ARFI. Using ARFI, manipulation of a probe, such as pushing to nodule, is unnecessary; therefore, measurement errors between examiners are low. In this study, nodule Vs of "X.XX m/s" showed either a "black" or "honeycomb" pattern on VTTI. Taking these results into consideration, nodules of papillary carcinomas had faster Vs and/or heterogeneous tissue structure; therefore, most of the nodules showed Vs of "X.XX m/s". At present, it is difficult to evaluate accurately the Vs of papillary carcinomas using an ACUSON $\mathrm{S}^{2000^{\circledR}}{ }^{\circledR}$, and quantitative statistical analysis of this series has never been accomplished. The improvement of device may reveal the Vs of papillary carcinomas more accurately.

The usability of VTTQ technology in differentiation in thyroid nodules has been reported. Using the same device (ACUSON S2000 ${ }^{\circledR}$ ), Gu et al. reported that different values of Vs were detected between benign adenomas $(2.03 \mathrm{~m} / \mathrm{s})$ and thyroid malignancies $(3.94 \mathrm{~m} / \mathrm{s})$ [10]. Concerning Vs in thyroid lesions, Zhang et al. reported $4.82 \mathrm{~m} / \mathrm{s}$ in papillary carcinomas, and $2.34 \mathrm{~m} / \mathrm{s}$ in benign nodules [11]. Bojunga et al. reported Vs of $2.69 \mathrm{~m} / \mathrm{s}$ in thyroid carcinomas, and of $1.90 \mathrm{~m} / \mathrm{s}$ in benign nodules [12]. These velocities showed a statistically significant difference and they concluded that ARFI imaging can be used as an additional tool in the diagnostic work-up of thyroid nodules.

In contrast to these reports, most papillary carcinomas showed Vs of "X.XX m/s" in the present study; nevertheless we used the same device. Lesions other than papillary carcinomas never showed Vs of "X.XX m/s". The reason for this difference is unclear. Concerning the quantitative measurement of Vs of breast cancer, most breast cancers showed Vs of "X.XX m/s"; however, measurement of margin of the tumor, Vs could be measured since this area involves normal mammary tissue $[13,14]$. Such Vs are defined as "marginal value", and if measuring marginal value, nodules of papillary carcinomas might be estimated. However, in this study, we planned to compare the quantitative elasticity and pathology of the same region accurately. So, we settled the ROI in the center of the nodules and pathological sections were made, similar to an ultrasound image. The same ROI was diagnosed pathologically. In addition, we also examined nodules' comparative elasticity using VTTI. Thus, our data investigated accurate Vs of nodules, although further study is needed. However, from our experience, papillary carcinomas showed higher elasticity compared to both normal region and other thyroid lesions. It is particularly important to note that nodule Vs showing "X.XX m/s" may suggest papillary carcinoma. Although data itself represents an error value, our preliminary report showed that measurements of Vs in thyroid nodules are beneficial in the diagnosis of papillary thyroid carcinoma.

\section{REFERENCES}

[1] Rago, T., Vitti, P., Chiovato, L., Mazzeo, S., De Liperi, A., Miccoli, P., Viacava, P., Bogazzi, F., Martino, E. and Pinchera, A. (1998) Role of conventional ultrasonography and color flow-doppler sonography in predicting malignancy in "cold" thyroid nodules. European Journal of Endocrinology, 138, 41-46. http://dx.doi.org/10.1530/eje.0.1380041

[2] Fukunari, N., Nagahama, M., Sugino, K., Mimura, T., Ito, K. and Ito, K. (2004) Clinical evaluation of color doppler imaging for the differential diagnosis of thyroid follicular lesions. World Journal of Surgery, 28, 1261-1265. http://dx.doi.org/10.1007/s00268-004-7597-8

[3] Ivanac, G., Brkljacic, B., Ivanac, K., Huzjan, R., Skreb, F. and Cikara, I. (2007) Vascularisation of benign and malignant thyroid nodules: CD US evaluation. Ultraschall in 
der Medizin, 28, 502-506. http://dx.doi.org/10.1055/s-2007-963023

[4] Bojunga, J., Herrmann, E., Meyer, G., Weber, S., Zeuzem, S. and Friedrich-Rust, M. (2010) Real-time elastography for the differentiation of benign and malignant thyroid nodules: A meta-analysis. Thyroid, 20, 1145-1150. http://dx.doi.org/10.1089/thy.2010.0079

[5] Asteria, C., Giovanardi, A., Pizzocaro, A., Cozzaglio, L., Morabito, A., Somalvico, F. and Zoppo, A. (2007) USelastography in the differential diagnosis of benign and malignant thyroid nodules. Thyroid, 18, 523-531. http://dx.doi.org/10.1089/thy.2007.0323

[6] Rubaltelli, L., Corradin, S., Dorigo, A., Stabilito, M., Tregnaghi A, Borsato, S. and Stramare, R. (2008) Differential diagnosis of benign and malignant thyroid nodules at elastosonography. Ultraschall in der Medizin, 30, 175-179. http://dx.doi.org/10.1055/s-2008-1027442

[7] Carneiro-Pla, D. (2013) Ultrasound elastography in the evaluation of thyroid nodules for thyroid cancer. Current Opinion in Oncology, 25, 1-5. http://dx.doi.org/10.1097/CCO.0b013e32835a87c8

[8] Sporea, I., Sirli, R., Bota, S., Vlad, M., Popescu, A. and Zosin, I. (2012) ARFI elastography for the evaluation of diffuse thyroid gland pathology: Preliminary results. World Journal of Radiology, 4, 174-178. http://dx.doi.org/10.4329/wjr.v4.i4.174

[9] Nigtingale, K., McAleavey, S. and Trahey, G. (2003) Shear-wave generation using acoustic radiation force: In vivo and ex vivo results. Ultrasound in Medicine \& Biology, 29, 1715-1723. http://dx.doi.org/10.1016/j.ultrasmedbio.2003.08.008

[10] Gu, J., Du, L., Bai, M., Chen, H., Jia, X., Zhao, J. and Zhang, X. (2012) Preliminary study on the diagnostic value of acoustic radiation force impulse technology for differentiating between benign and malignant thyroid nodules. Journal of Ultrasound in Medicine, 31, 763-771.

[11] Zhang, Y.F., Xu, H.X., He, Y., Liu, C., Guo, L.H., Liu, L.N. and Xu, J.M. (2012) Virtual touch tissue quantification of acoustic radiation force impulse: A new ultrasound elastic imaging in the diagnosis of thyroid nodules. PLoS One, 11, e49094. http://dx.doi.org/10.1371/journal.pone.0049094

[12] Bojunga, J., Dauth, N., Berner, C., Meyer, G., Holzer, K., Voelkl, L., Herrmann, E., Schroeter, H., Zeuzem, S. and Friedrich-Rust, M. (2012) Acoustic radiation force impulse imaging for differentiation of thyroid nodules. PLoS One, 7, e42735. http://dx.doi.org/10.1371/journal.pone.0042735

[13] Tozaki, M., Isobe, S. and Fukuma, E. (2011) Preliminary study of ultrasonographic tissue quantification of the breast using the acoustic radiation force impluse (ARFI) technology. European Journal of Radiology, 80, e182e187. http://dx.doi.org/10.1016/j.ejrad.2011.05.020

[14] Tozaki, M., Isobe, S. and Sakamoto, M. (2012) Combination of elastography and tissue quantification using the acoustic radiation force impulse (ARFI) technology for differential diagnosis of breast masses. Japanese Journal of Radiology, 30, 659-670. http://dx.doi.org/10.1007/s11604-012-0106-3 